Thorax, 1979, 34, 276-278

\title{
Traumatic rupture of a retro-oesophageal aortic arch with congenital vascular ring
}

\author{
PIERLUIGI de MOZZI, UBERTO BORTOLOTTI, DINO CASAROTTO, DARIO BETTI, \\ ALBERTO FRACASSO, AND VINCENZO GALLUCCI
}

From the Department of Cardiovascular Surgery, University of Padova Medical School, Padova, Italy

Traumatic rupture of the aorta at the level of the arch is an exceptionally rare lesion (Charles et al, 1977). To our knowledge no report of a traumatic rupture of a retro-oesophageal aortic arch, in a patient with a congenital vascular ring, has been published.

\section{Case report}

A 21-year-old man suffered a closed chest injury on 6 August 1977 in a motor accident. On admission to the intensive care unit of an out-oftown hospital the patient showed some clinical signs of shock: his blood pressure was $85 / 65$ $\mathrm{mmHg}$ and the pulse rate 112 and regular. He was conscious but disorientated and complained of severe retrosternal chest pain. There were no abnormal neurological signs.

Radiographic studies showed a widened mediastinum and a fracture of the right tibia. As a traumatic rupture of the thoracic aorta was suspected he was transferred to our department, where an angiographic study was immediately performed. This showed a rupture of the aortic arch and defective filling of the brachiocephalic vessels (fig 1).

As the patient's condition was rapidly deteriorating, he was taken to the operating theatre, where a median sternotomy was performed. Cardiopulmonary bypass was available. After the pericardial sac had been opened, direct inspection showed a previously unsuspected retro-oesophageal position of the aortic arch, which was obviously the site of the laceration. The adventitia was still intact. The brachiocephalic vessels were abnormal in origin. The first branch of the arch was the left carotid artery, next arose the right carotid artery, and lastly the right subclavian artery originated from the posterior surface of the distal ascending aorta (fig 2).

As it appeared impossible to reach the aortic tear through this approach, the sternum was closed and a left posterolateral thoracotomy in the

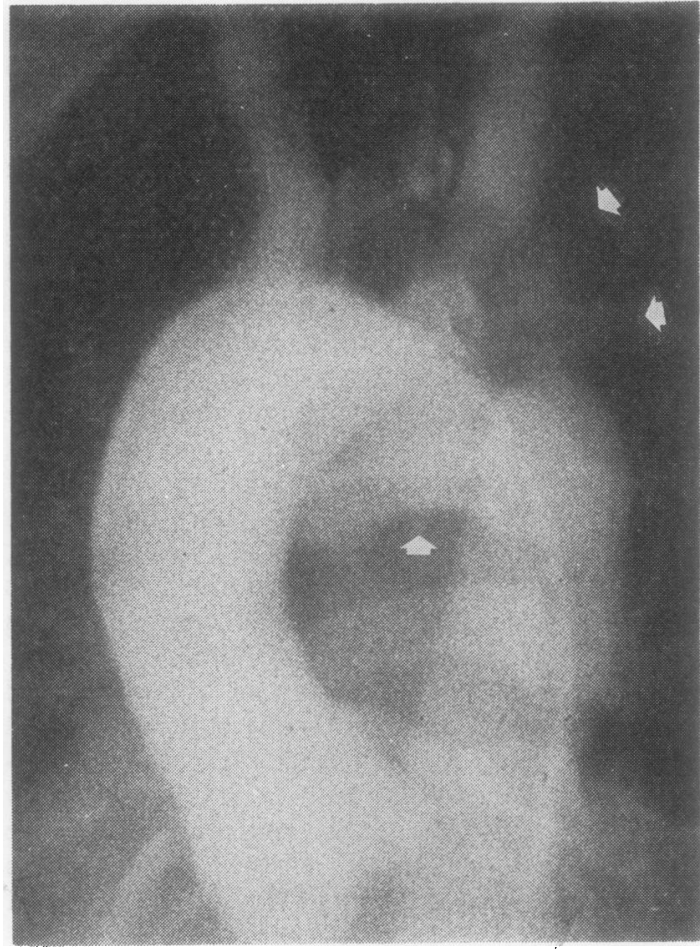

Fig 1 Preoperative aortogram. Arrows indicate site of aortic laceration.

fourth intercostal space was performed. The left chest contained about $1000 \mathrm{ml}$ of blood. A large mediastinal haematoma was affecting the retrooesophageal aortic arch. The left subclavian artery appeared as a cord-like structure originating laterally to the oesophagus. A remnant of the ductus arteriosus was also present, and together with the arch and the left pulmonary artery completed the vascular ring.

The aorta was cross-clamped proximally to the origin of the right subclavian artery and distally below the origin of the left subclavian artery, 

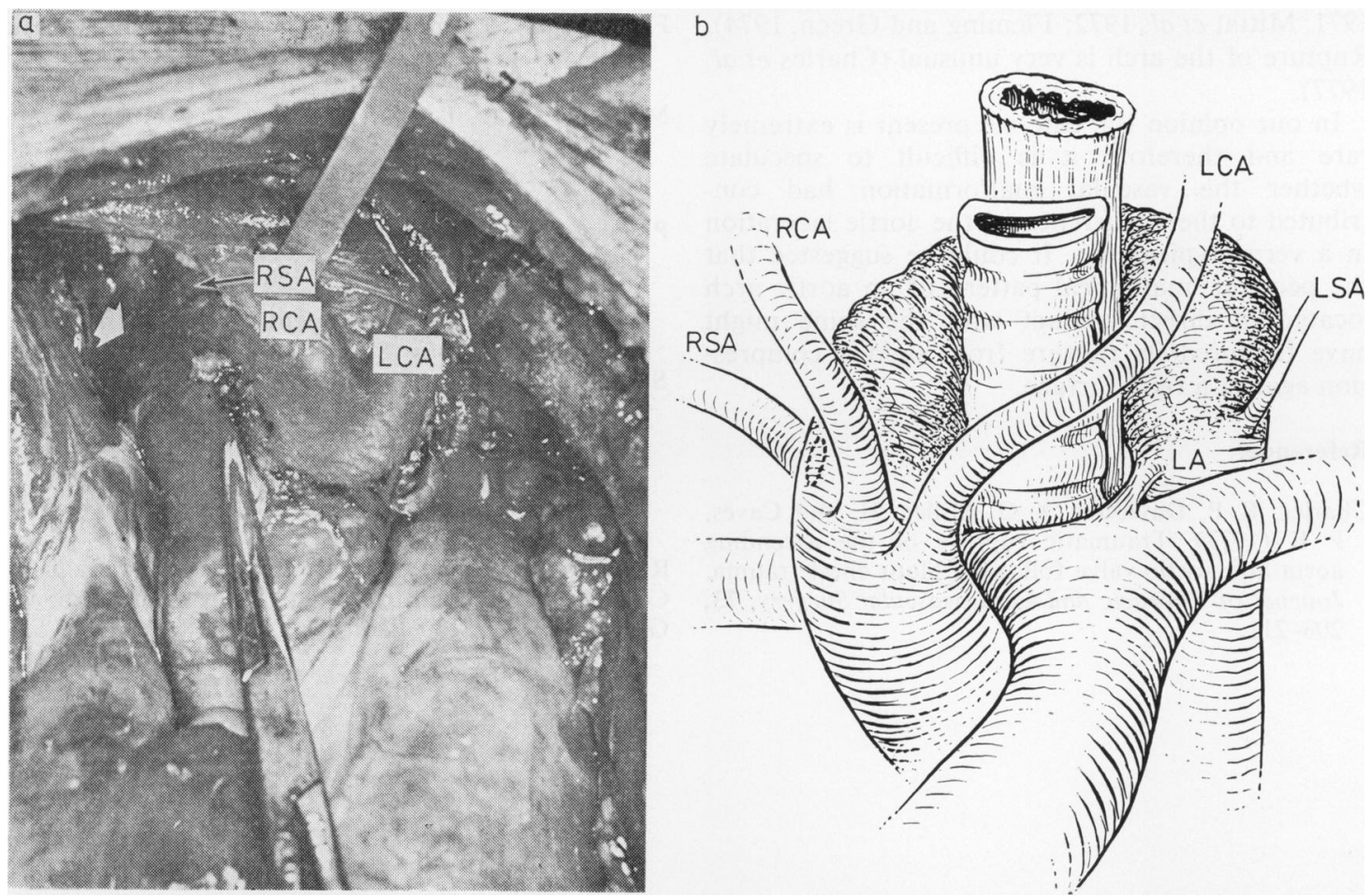

Fig 2 (a) Intraoperative view through a median sternotomy. Anomalous origin of brachiocephalic vessels is shown. Arrow indicates aortic haematoma. (b) Schematic drawing of anomalous anatomical pattern. $L C A=$ Left carotid artery; $R C A=$ Right carotid artery; $R S A=R i g h t$ subclavian artery; $L S A=$ Left subclavian artery; $L A=$ Ligamentum arteriosum.

which was ligated and divided. The circumferential aortic tear, affecting all parietal layers, was then repaired, and the aortic arch replaced with a $20 \mathrm{~mm}$ woven Dacron graft.

The postoperative period was uncomplicated, and the patient was discharged to the ward on the fourth day.

Three days later, despite adequate antibiotic treatment, he developed fever, probably caused by a right lower lobe pneumonia. Septicaemic shock developed, and blood cultures grew Escherichia coli. Specific antibiotic treatment was started, but death occurred on the 11th postoperative day. The necropsy confirmed that death was due to septic shock.

\section{Discussion}

A complete vascular ring consists of an anatomical continuity of vascular structures or their remnants around the trachea and the oesophagus. This is found in double aortic arch, in ductus arteriosus originating from the contralateral pulmonary artery, either isolated or associated with an anomalous subclavian artery, or when a left pulmonary artery runs behind the trachea (Shuford and Sybers, 1974).

The symptoms depend on the compression of the anatomical structures surrounded by the ring: if the ring is relatively wide the patient may be asymptomatic for a long time. In our patient the malformation was discovered only at operation, and he had no history of relevant symptoms.

The need to change the surgical approach during the operation created some additional problems for the surgeon, but nevertheless the procedure was successfully accomplished. Cardiopulmonary bypass was not employed, and in the postoperative period no neurological complications were observed.

There is general agreement that the aortic isthmus is the commonest site of rupture after closed chest trauma, while less of ten the ascending and descending aorta are affected (Piwnica et al, 
1971; Mittal et al, 1972; Fleming and Green, 1974). Rupture of the arch is very unusual (Charles et al, 1977).

In our opinion the case we present is extremely rare and therefore it is difficult to speculate whether the vascular malformation had contributed to the occurrence of the aortic laceration in a very atypical site. It could be suggested that the peculiar anatomical pattern of an aortic arch located in direct contact with the spine might have favoured its rupture from sudden compression against a rigid surface.

\section{References}

Charles, K P, Davidson, K G, Miller, H, and Caves, $P$ K (1977). Traumatic rupture of the ascending aorta and aortic valve following blunt chest trauma. Journal of Thoracic and Cardiovascular Surgery, 73, 208-211.
Fleming, A W, and Green, D C (1974). Traumatic aneurysm of the thoracic aorta. Report of 43 patients. Annals of Thoracic Surgery, 18, 91-101.

Mittal, A, May, I A, and Samson, P C (1972). Traumatic aneurysm of the aortic arch. Report of an unusual location. Annals of Thoracic Surgery, 13, 494-498.

Piwnica, A H, Chetochine, F, Soyer, R, and Winckler, C (1971). Traumatic rupture of the aortic arch with disinsertion of the innominate artery. Report of a case with successful treatment. Journal of Thoracic and Cardiovascular Surgery, 61, 246-252.

Shuford, W H, and Sybers, R G (1974). The Aortic Arch and its Malformations. Charles C. Thomas, Springfield, Ill.

Requests for reprints to: Professor V Gallucci, Centro di Cardiochirurgia, Università degli Studi, via Giustiniani 2, 35100 Padova. 